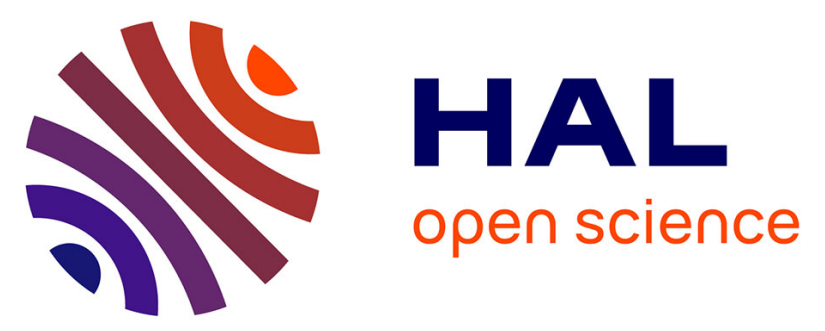

\title{
A Novel LQR-Based Cascaded Control Scheme of a Powered Knee Joint Orthosis for Rehabilitation
}

\author{
Ines Jammeli, Ahmed Chemori, Salwa Elloumi, Samer Mohammed
}

\section{To cite this version:}

Ines Jammeli, Ahmed Chemori, Salwa Elloumi, Samer Mohammed. A Novel LQR-Based Cascaded Control Scheme of a Powered Knee Joint Orthosis for Rehabilitation. ICSC 2021 9th International Conference on Systems and Control, Nov 2021, Caen, France. pp.361-366, 10.1109/ICSC50472.2021.9666730 . lirmm-03523073

\section{HAL Id: lirmm-03523073 https://hal-lirmm.ccsd.cnrs.fr/lirmm-03523073}

Submitted on 12 Jan 2022

HAL is a multi-disciplinary open access archive for the deposit and dissemination of scientific research documents, whether they are published or not. The documents may come from teaching and research institutions in France or abroad, or from public or private research centers.
L'archive ouverte pluridisciplinaire $\mathbf{H A L}$, est destinée au dépôt et à la diffusion de documents scientifiques de niveau recherche, publiés ou non, émanant des établissements d'enseignement et de recherche français ou étrangers, des laboratoires publics ou privés. 


\title{
A Novel LQR-Based Cascaded Control Scheme of a Powered Knee Joint Orthosis for Rehabilitation
}

\author{
Ines Jammeli ${ }^{1}$, Ahmed Chemori ${ }^{2}$, Salwa Elloumi ${ }^{1}$ and Samer Mohammed ${ }^{3}$
}

\begin{abstract}
In order to improve the healthcare services quality, various initiatives have been carried out to develop new assistive technologies such as wearable robots. This paper deals with the control of an actuated knee joint orthosis dedicated to assistive and rehabilitation purposes. The proposed control scheme is based on a nonlinear state feedback complied with a linear quadratic regulator (LQR) in a cascaded control architecture. The proposed control scheme has been validated in simulation for the control of an active orthosis in various operating conditions and compared with a PID controller. The obtained simulation results show clearly the efficiency of the proposed control scheme and its superiority with respect to the PID in terms of the tracking performance and the comfort of the user.
\end{abstract}

Index Terms - Wearable robot, Linear quadratic regulator, Linearization, knee joint impairments, rehabilitation.

\section{INTRODUCTION}

The increasing number of people living with physical disability has become a global socioeconomic issue and this is largely due to the rising ageing rate. Elderly people with weakened muscle strength may lose their stability during walking, thus they require the help of other people. The daily living tasks could become really challenging for people with motor impairment. This brings considerable attention on how to provide assistance for the elderly as well as people with lower and/or upper limb pathologies in their daily life, especially concerning mobility and autonomy [3]. To reduce the burden on care services, many initiatives have been set up to promote research on developing assistive care technologies. With recent technological advances, physical assistive robotics is emerging as a promising solution for developing systems to facilitate and improve the daily life conditions for people with reduced mobility. In particular, wearable robots have gained great attention in the last decade for applications related to rehabilitation, assistance, and human capabilities augmentation. Wearable robots are mechatronic devices embodied by the human upper and/or lower limbs that are equipped with sensors and actuators. They are intended to strengthen human physical abilities in the upper/lower limbs, to support people with reduced mobility in daily living activities and to automatically rehabilitate human joints and muscles enabling the recovery

\footnotetext{
${ }^{1}$ Ines Jammeli and Salwa Elloumi are with LSA, EPT, University of Carthage, ENICarthage Tunis, Tunisia. jammeli.ines@gmail.com, salwa.elloumi@gmail.com

${ }^{2}$ Ahmed Chemori is with LIRMM, University of Montpellier, CNRS, Montpellier, France. Ahmed. Chemorielirmm. fr

3 Samer Mohammed is with LISSI, Paris-Est Créteil University, Paris, France. samer.mohammed@u-pec.fr
}

and the enhancement of the user's limb control [1]-[2]

Over the previous few decades, several exoskeletons have been developed and commercialized. The BLEEX, is one of the first motorized lower-limb exoskeleton prototype designed to decrease metabolic consumption, reduce the risk of back and leg injuries, and improve the capabilities of soldiers, firefighters, and rescuers when transporting heavy loads over long distances and in difficult situations. Force sensors are fixed under the soles of both feet, and actuations are performed at the hip, knee, and ankle. This exoskeleton, with seven degrees-of-freedom (DoF) at each leg, adopts a hybrid hydraulic-electric portable power supply [4]. The controller described in [5] uses the inverse dynamics of the exoskeleton as a positive feedback controller. The RoboKnee is a single-degree-of-freedom lower-limb exoskeleton that acts on the knee joint to assist human movement. It allows the wearer to climb stairs and do deep knee bends while handling heavy load in their backpack. The intention of the user is determined through the knee joint angle and ground reaction forces. RoboKnee employs a hierarchical control strategy, which uses a straightforward mid-level force generation scheme based on a positive force feedback amplification coupled with a low-level closed-loop force based on a PD control loop [6]-[7]. The ReWalk is an active lower limbs orthosis attached to the patient's legs, that allows paraplegics to stand, sit, and walk without assistance. A wireless remote control worn on the wrist allows the patient to select the activity he/she wants to perform. The ReWalk hip and knee joints are powered and guided along a predefined path [3]. The stability of the user during walking is ensured by crutches. The device, which is controlled by DC motors at the joints, is customized and sized for each patient [10]. The Hybrid Assistive Limb (HAL) is another well-known commercialized exoskeleton. It is a four-degree-of-freedom bipedal locomotion system designed to help elderly and paraplegics with daily tasks such as sit-to-stand and stand-to-sit. Its movements are based on the use of EMG (Electromyography) sensors to detect motion intention. Moreover, the hybrid lower limb determines the wearer's center of pressure using floor response force sensors to ensure his/her stability. Each joint of the HAL system is driven by a PD controller [11]. There are also treadmill-based exoskeletons designed for rehabilitation and assistance of people with reduced mobility, such as the Lokomat. This latter is a powered gait orthosis that automates and assists walking motions, allowing more efficient treadmill training and less physically demanding rehabilitation. This therapeutic tool, which is 
controlled by DC actuators at the knee and hip, is used to treat patients with cerebrovascular accident, spinal cord injury, traumatic brain injury, Parkinson disease or multiple sclerosis. Lokomat is only used in clinical training sessions that are tailored to each patient's particular needs in terms of desired models and duration [8]-[9].

The control of the EICOSI (Exoskeleton The control of the EICOSI (Exoskeleton Intelligently COmmunicating and Sensitive to Intention) system using Linear Quadratic Regulation, is the subject of our research study. This prototype was developed at the Lissi Laboratory of University Paris-Est Créteil in France. It is an active lower limb orthosis with one degree of freedom that acts on the knee joint level, designed for assistive and rehabilitation purposes. Several controls, including PID, sliding mode control, and adaptive control, have been applied to EICOSI. In this paper, we propose to control it using an exact input output liearization feedback and an LQR control. The LQR appears to be a good choice in our case since it has been proven that good tracking of the therapist's trajectory accelerates patient recovery and that the robustness and stability of the controller are required when dealing with robots in direct interaction with humans. Since PID controllers are commonly used in academia and industry, the results of simulations using the proposed approach were compared to those obtained using a PID controller. The shank foot was freely moving around the knee joint with no interaction with the ground.

The following is a breakdown of the paper's structure: the EICOSI active lower limb orthosis is described in Section II. The suggested LQR control method is described in Section III. Section IV covers the simulation results of flexion/extension movements performed on a healthy subject in a sitting position with no contact with the ground, as well as a comparison study with a PID controller to illustrate the efficiency of our approach. Some conclusions and future scopes are drawn in section $\mathrm{V}$.

\section{DESCRIPTION OF THE EICOSI ACTIVE ORTHOSIS}

In this study, the EICOSI active orthosis was used to provide power assistance at the user's knee joint level for flexion/extension exercises. The orthosis illustrated in Fig. 1, is driven using a high-power brushless DC motor (Maxon, Switzerland). A small transmission system with a gear motor, a ball screw, a transmission belt, and a cable drive which are designed to ensure an efficient and portable system with a relatively high output torque (up to approximately 18 N.m). The brushless DC motor is equipped with an incremental encoder that measures the motor rotation angle and serves to compute the knee joint angle. A numerical derivative of the measured joint angle yields the angular velocity [13]-[14].

This 1 DoF active orthosis allows the user to conduct flexion and extension motions with a relative angle ranging from $0^{\circ}$ to $135^{\circ}, 0^{\circ}$ for full knee joint-orthosis extension, $135^{\circ}$ for maximum knee joint-orthosis flexion, and $90^{\circ}$ for resting position. The studied system represents a person

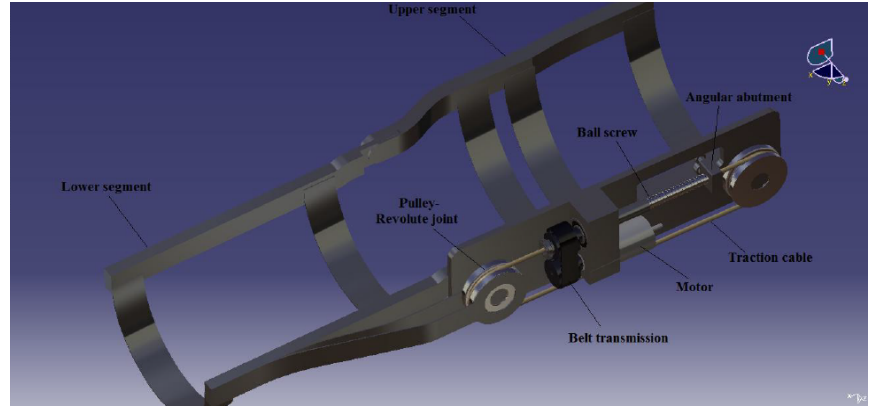

Fig. 1. View of EICOSI active lower limb orthosis.

in a sitting position with no interaction with the ground, wearing the actuated orthosis consisting of two segments attached separately to the thigh and shank of the wearer using appropriate braces. The shank-foot of the wearer is considered as a single rigid segment and can freely move around the knee joint. The entire system (human shank and embodied actuated orthosis) is illustrated by the following second-order equation:

$J \ddot{\theta}(t)=\tau_{g} \cos (\theta(t))-f_{s} \operatorname{sign}(\dot{\theta}(t))-f_{v} \dot{\theta}(t)+u(t)+\tau_{h}(t)$

where $\theta, \dot{\theta}$, and $\ddot{\theta}$ are respectively the angular position, velocity and acceleration, $f_{s}$ and $f_{v}$ are the solid and viscous friction coefficients, and $J$ is the inertia of the whole system (the active orthosis and its wearer lower limb). $\tau_{g}, \tau_{h}$ and $u$ are respectively the gravitational torque, the human's torque, and the input applied to the system [15]-[20].

\section{LQR CONTROLLER DESIGN FOR THE EICOSI ORTHOSIS}

The linear quadratic regulator is an optimal control theorybased feedback controller:

$$
u(t)=-K\left(x(t)-x_{r e f}(t)\right)
$$

It computes the feedback gain matrix $K$ using the state space representation of the controlled system and the weighting matrices $Q$ and $R$ supplied by the user [21]-[22]:

$$
\left\{\begin{array}{l}
\dot{x}(t)=A x(t)+B u(t) \\
y(t)=C x(t)
\end{array}\right.
$$

where $A$ is the state matrix, $B$ is the input matrix, and $C$ is the output matrix. The cost function that collects the mathematically defined target performances is minimized, whether on an infinite or finite horizon (depending on the user's preference). The used infinite horizon cost function is illustrated by the following equation (4):

$$
V(t)=\int_{0}^{\infty}\left[\left[x(t)-x_{r e f}(t)\right]^{T} Q\left[x(t)-x_{r e f}(t)\right]+u^{T}(t) R u(t)\right] d t
$$


The gain matrix $K$ of the feedback control law that minimizes $V(t)$ is defined by equation (5):

$$
K=R^{-1} B^{T} P
$$

where $P$ is calculated by solving the continuous time algebraic Riccati equation (6):

$$
A^{T} P+P A-P B R^{-1} B^{T} P+Q=0
$$

As it can be seen from the equations (5) and (6), the weighting matrices $Q$ and $R$ intervene in the definition of the gain matrix $K$. How to choose these matrices $(Q$ and $R$ ) is one of the most important concerns in LQR design. In general, they are chosen as diagonal matrices in order to simplify the tuning. We can assign greater weight values to states that should be kept tiny using such matrices. Similarly, we can penalize an entry against states and other entries by adjusting its corresponding weight [23]-[24].

$$
Q=\left(\begin{array}{ccc}
q_{1} & & 0 \\
& \ddots & \\
0 & & q_{n}
\end{array}\right), R=\left(\begin{array}{ccc}
r_{1} & & 0 \\
& \ddots & \\
0 & & r_{m}
\end{array}\right)
$$

Since the LQR is a model-based controller, the shankorthosis parameters were obtained using a nonlinear least square optimization method. The regression equations of Winter were used to compute the shank mass and the gravity center, taking into consideration the subject's weight and height and the remaining parameters were identified by a passive pendulum test. The acquired parameters illustrated in the TABLE I, are used in the simulation scenarios presented in section IV. We used the exact input-output linearization

TABLE I

SUMMARY OF THE DYNAMIC PARAMETERS OF THE SYSTEM.

\begin{tabular}{ccc} 
Parameter & Symbol & Value \\
\hline Solid friction coefficient & $f_{s}$ & $0.4069 \mathrm{~N} . \mathrm{m}$ \\
Viscous friction coefficient & $f_{v}$ & $2.3708 \mathrm{~N} . \mathrm{m} . \mathrm{s} . \mathrm{rad}^{-1}$ \\
Inertia & $J$ & $0.256 \mathrm{Kg} . \mathrm{m}^{2}$ \\
Gravity torque & $\tau_{g}$ & $3.4689 \mathrm{N.m}$ \\
\hline
\end{tabular}

method in order to be able to apply the LQR with our nonlinear system as illustrated in Fig. 2. Through a change of variables and appropriate nonlinear feedback, the nonlinear system can be transformed into a linear equivalent one as shown in the state space representation below:

$$
\left\{\begin{array}{l}
\dot{x}(t)=A x(t)+B v(t) \\
y(t)=C x(t)
\end{array}\right.
$$

with $A=\left(\begin{array}{ll}0 & 1 \\ 0 & 0\end{array}\right), \mathrm{B}=\left(\begin{array}{l}0 \\ 1\end{array}\right)$ and $\mathrm{C}=\left(\begin{array}{ll}1 & 0\end{array}\right)$

As it can be seen from (8), a linear input-output map was established between the new input $v(t)$ and the output $y(t)$ by considering the following change of variables:

$u(t)=J v(t)-\tau_{g} \cos (\theta)(t)+f_{s} \operatorname{sign}(\dot{\theta}(t))+f_{v} \dot{\theta}(t)-\tau_{h}(t)$

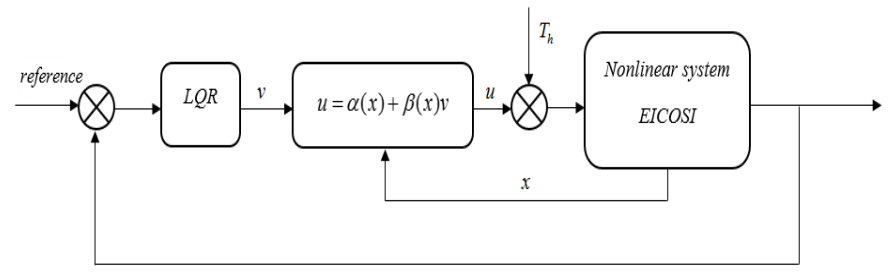

Fig. 2. Block diagram of the proposed control scheme applied to EICOSI orthosis.

\section{Simulation Results : A COMPARison BetweEN PID AND LQR CONTROLLERS}

In this section, the simulation results that were performed to evaluate the LQR controller's performances while comparing them to those of a PID controller, are described and analyzed. This latter is tuned through trial and error method until achieving the best settling time, precise tracking, and stability. The simulation results are performed in the case of a sitting position with no interaction with the ground. Four separate scenarios are used to validate the proposed LQR control approach. The considered reference trajectory in the first case is configured to a chirp signal in passive mode while it is equal to a square wave used for flexion and extension movements, in the second scenario. Unlike the two previous scenarios, the torque $\tau_{h}$ is taken into consideration in the two last ones. During scenario 3 , the human torque is considered as white noise while a human walking cycle trajectory is used as reference. During the last scenario, $\tau_{h}$ is used to represent the assistive (the orthosis user is delivering an assistance effort) and resistive modes (the subject is delivering an effort in the opposite direction of the input control $u(t)$ ), while a sinusoidal input signal with constant frequency and amplitude is used as a reference trajectory.

\section{Scenario 1: Passive Mode - Chirp reference trajectory}

During this scenario, $\tau_{h}$ is considered null. The reference trajectory is taken equal to a chirp signal. The goal of this scenario is to see if the controllers are able to track the reference trajectory as it accelerates. The PID controller's curves are depicted in blue, the LQR controller's curves are depicted in black, while the reference curves are depicted in red. The absolute values of the tracking errors for angular positions and velocities, illustrated by curves 2 and 4 in Fig. 3 , indicate that the PID's tracking errors are significantly greater than the LQR ones while the delivered torques are approximately the same. The RMSEs (Root Mean Square Errors), whose formula is given by equation (10), are calculated and compared in both situations to corroborate these results.

$$
R M S E=\sqrt{\frac{\sum_{i=1}^{n}\left(z_{r e f}(i)-z(i)\right)^{2}}{n}}
$$

with $n$ : the number of the considered samples and $z$ either representing the angular position or the angular velocity. 
In the case of the LQR, the angular position RMSE is $1.3710^{-6} \mathrm{rad}$, while in the case of the PID, it is $5.2110^{-5} \mathrm{rad}$. These results confirm the LQR's superiority over the PID controller. In terms of angular velocity RMSE computation, we found that the value of the LQR RMSE was equal to $7.710^{-6}(\mathrm{rad} / \mathrm{s})$, whereas the value for the PID was equal to $1.0610^{-4}(\mathrm{rad} / \mathrm{s})$. These calculations back up the observations made based on curves shown in Fig. 3.
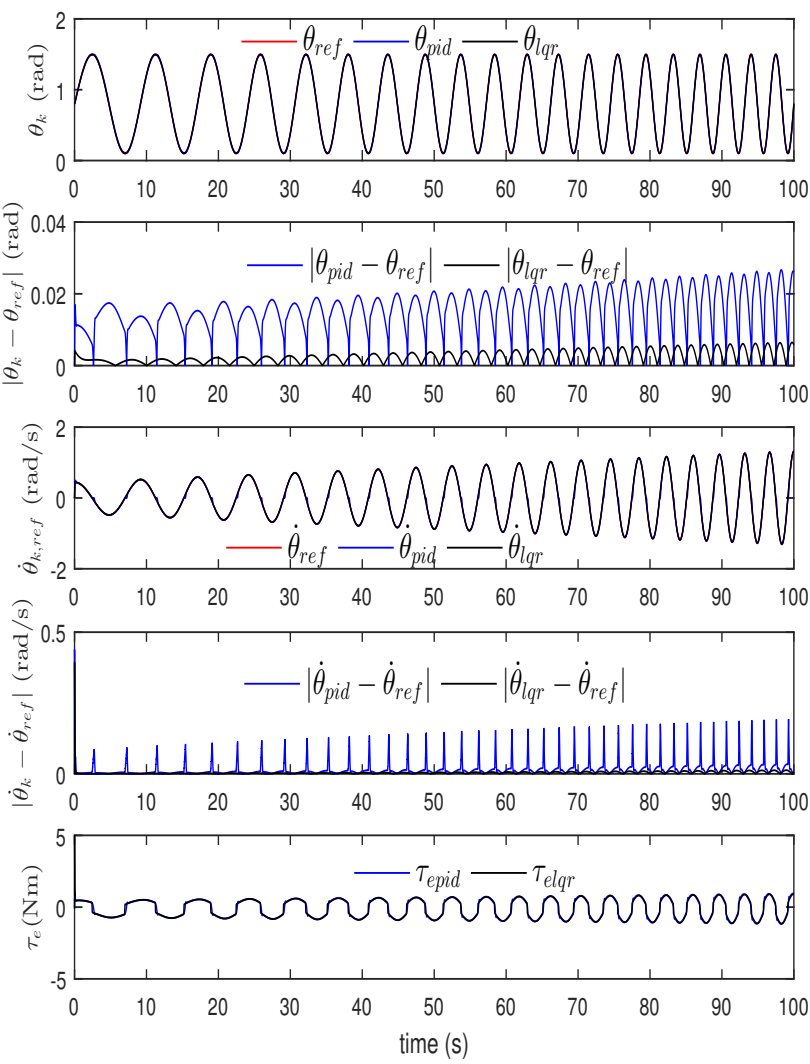

Fig. 3. Scenario 1: PID/LQR controllers : null $\tau_{h}$ torque with a chirp reference trajectory.

\section{Scenario 2: Passive Mode - Square reference trajectory}

To illustrate the flexion/extension exercise performed for the rehabilitation of patients with reduced mobility, we use a reference trajectory equivalent to a square signal. During this scenario, the torque $\tau_{h}$ is also considered null. The curves depicted in Fig. 4 show that the LQR performs better than the PID in this scenario as well. Indeed, the LQR allowed for better trajectory tracking without resorting to overshoot while delivering lower torque values compared to the PID controller. It can be observed that the PID has reached the motor's maximum values, resulting in saturation, according to the zoom performed around time 20s. Repeatedly engaging in such activity can damage the active orthosis motor.

Scenario 3: Passive Mode with white noise - Human walking cycle reference trajectory
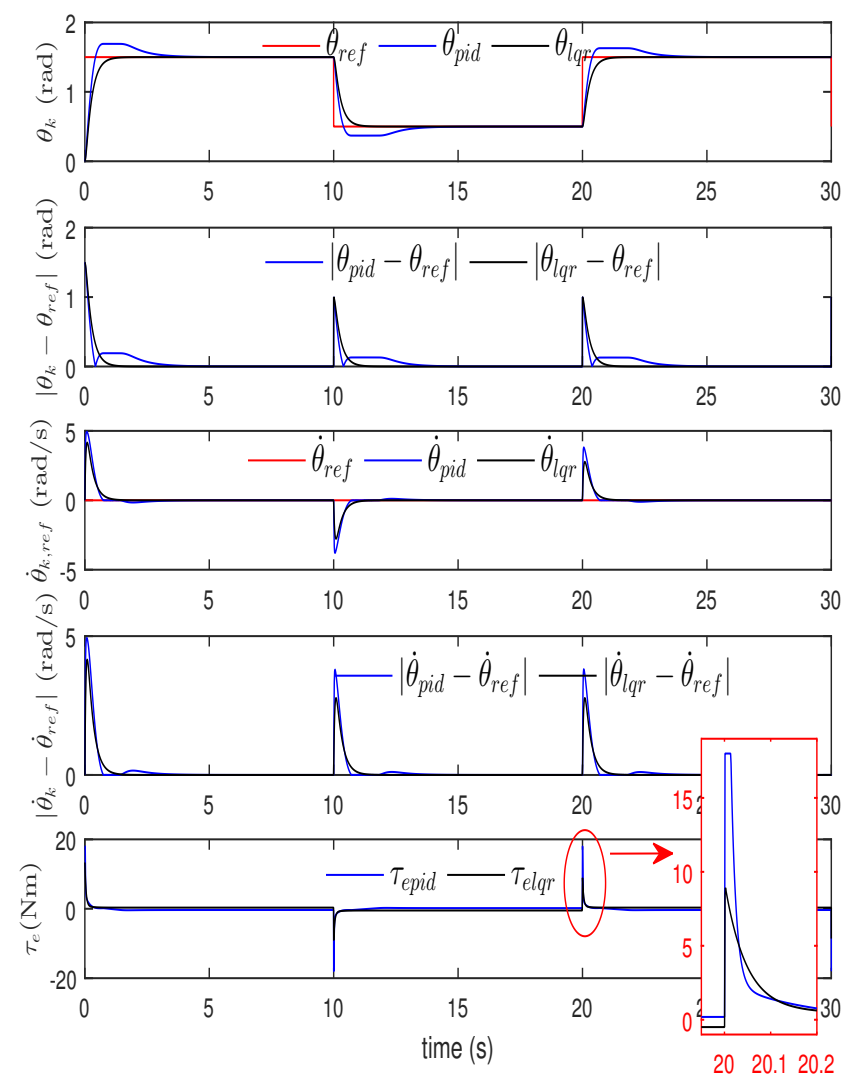

Fig. 4. Scenario 2: PID/LQR controllers : no effort exerted by the patient - square reference trajectory.

The reference trajectory used in this scenario is the human walking cycle. The orthosis wearer is considered to be passive, however to simulate real experimental conditions, we set $\tau_{h}$ equal to a white noise. The two controllers were able to reject the noise caused by the human torque, as demonstrated by the curves in Fig. 5. Although the torques generated by the LQR and the PID are nearly identical, the LQR's trajectory tracking in terms of angular position and angular velocity is far superior to the PID tracking. Curves 2 and 4 in Fig. 5 clearly illustrate this observation. For the two controllers, the computed RMSEs yielded to the following values $4.1510^{-4}(\mathrm{rad})$ and $2.510^{-3}(\mathrm{rad} / \mathrm{s})$ for PID and $1.5410^{-5}$ (rad) and $7.8710^{-5}(\mathrm{rad} / \mathrm{s})$ for LQR. This scenario reveals once again the superiority of LQR over PID.

Scenario 4: Passive Mode / Assistive Mode / Resistive Mode - A sinusoidal reference trajectory with a constant frequency.

The reference trajectory in this scenario (shown in Fig. 6) is sinusoidal with constant frequency and amplitude. The $\tau_{h}$ torque is also taken into consideration. We consider the passive mode for the first 30 seconds, where $\tau_{h}$ is zero then we set it to an assistive effort between 30s and $60 \mathrm{~s}$. In this scenario, we want to see if the controllers 

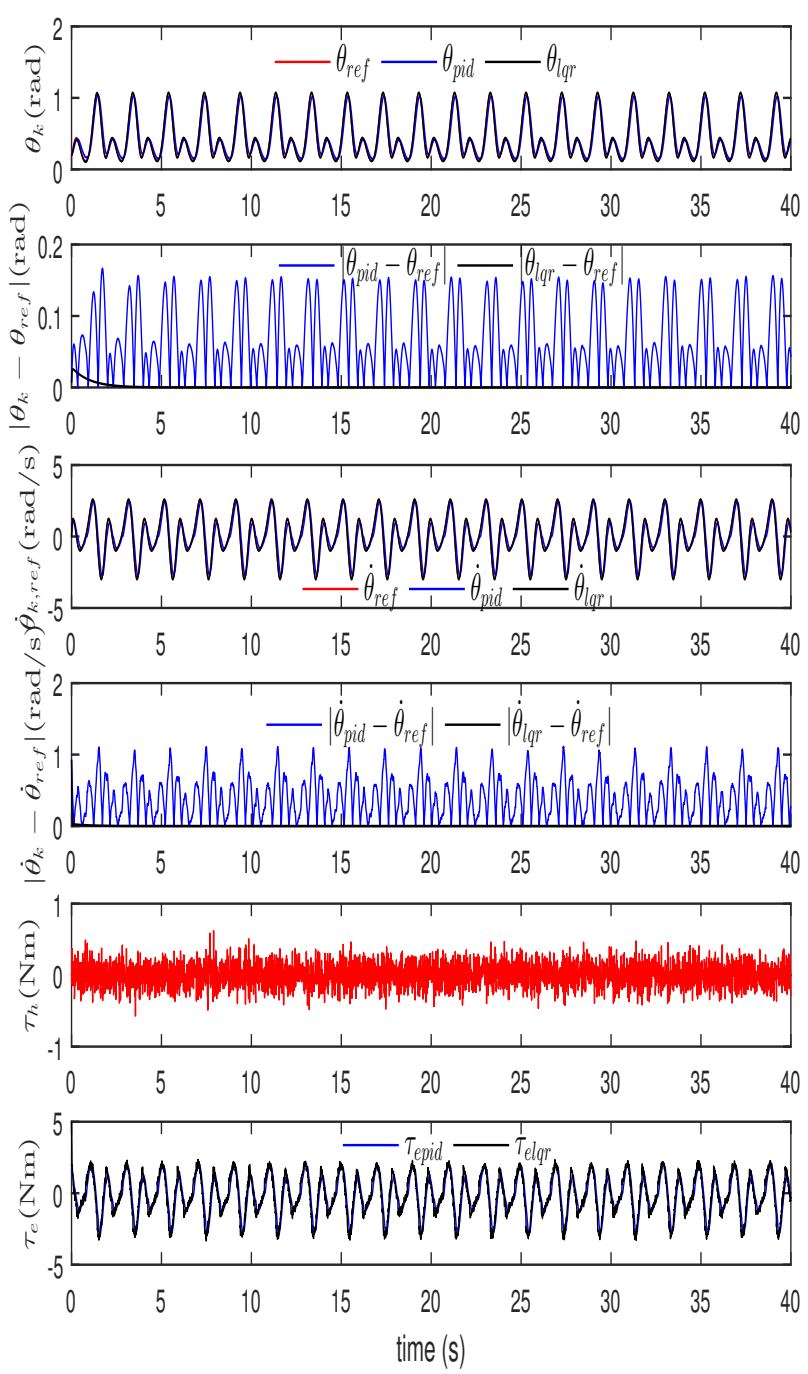

Fig. 5. Scenario 3: PID/LQR controllers : no effort exerted by the patient - human walking cycle reference trajectory.

can compensate for human torque and assist the person only when needed. During the rehabilitation phase, this behavior is important, and should be considered while designing a wearable robot. In fact, involving the patient in rehabilitation exercises can help the user heal faster. To illustrate a resistive force, $\tau_{h}$ is taken equal to a force exerted in the opposite direction of the torque delivered by the orthosis during the last 30 seconds. Torques generated in both cases are significantly reduced in assistive mode, allowing reference trajectory tracking when the human is expected to provide an assistance force, whereas torques generated in resistive mode are larger, allowing the subject to compensate for resistance force. The LQR controller is able to compensate for the required torque during the final phase, resulting in better performances than the PID.

We gathered the computed RMSEs during the four scenarios in one table, to make comparisons between the two controllers easier. We can clearly see that the
LQR controller surpasses the PID controller in terms of position tracking error and angular velocity smoothness almost during all scenarios as shown in TABLE II. This smoothness makes the active orthosis more comfortable to wear and offers more protection to the human leg.
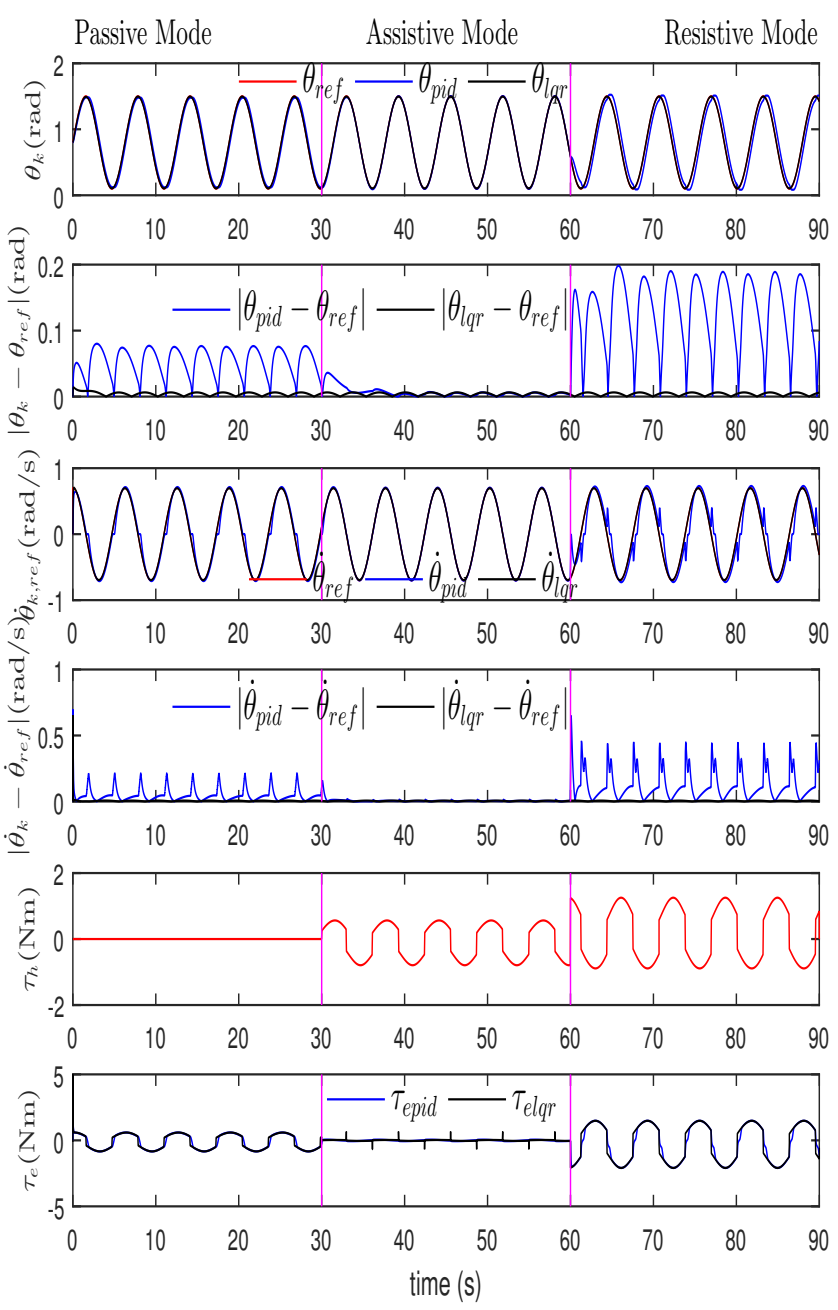

Fig. 6. Scenario 4: PID/LQR controllers : no effort during the first 30 s, exerting an assistive effort between $30 \mathrm{~s}$ and $60 \mathrm{~s}$ and exerting a resistive effort during the last $30 \mathrm{~s}$.

TABLE II

SUMMARY OF THE OBTAINED RMSE'S.

\begin{tabular}{ccccc} 
Scenario & $\begin{array}{c}P I D_{R M S E_{\theta}} \\
(\mathrm{rad})\end{array}$ & $\begin{array}{c}L Q R_{R M S E_{\theta}} \\
(\mathrm{rad})\end{array}$ & $\begin{array}{c}P I D_{R M S E_{\dot{\theta}}} \\
(\mathrm{rad} / \mathrm{s})\end{array}$ & $\begin{array}{c}L Q R_{R M S E_{\dot{\theta}}} \\
(\mathrm{rad} / \mathrm{s})\end{array}$ \\
\hline 1 & $5.2110^{-5}$ & $9.2910^{-6}$ & $1.0610^{-4}$ & $1.7610^{-5}$ \\
2 & $8.2410^{-4}$ & $8.8410^{-4}$ & $3.610^{-3}$ & $2.810^{-3}$ \\
3 & $4.1510^{-4}$ & $1.5410^{-5}$ & $2.510^{-3}$ & $7.8710^{-5}$ \\
4 & $2.8910^{-4}$ & $1.5610^{-5}$ & $3.5210^{-4}$ & $2.8710^{-5}$ \\
\hline
\end{tabular}

\section{CONCLUSION AND FUTURE WORK}

In this paper, a linear quadratic regulator was used to control the EICOSI exoskeleton, a one degree-of-freedom active orthosis acting on the knee joint, used for assistive and rehabilitation purposes. Since the studied system 
is stated as a nonlinear second order model, an exact input-output linearization feedback was performed, allowing the application of the LQR controller on the knee active orthosis. Following the results of four separate experimental scenarios, a comparison between simulation results achieved while using the PID controller and the LQR controller was developed and detailed. Hence, the results confirm that the LQR outperforms the PID controller in terms of position tracking error, energy consumption, and smoothness of the resulting angular velocity. Executing these experiments with distinct scenarios on subjects wearing the active orthosis in a sitting position will be the next stage for this research.

\section{REFERENCES}

[1] B. Chen, H. Ma, L.Y Qin, F. Gao, K.M Chan, S.W Law, L. Qin and W.H Liao, Recent developments and challenges of lower extremity exoskeletons, Journal of Orthopedic Translation 5, 26 - 37, 2016.

[2] World Health Organization: "Ageing and health", http://www.who.int/news-room/fact-sheets/detail/ageing-andhealth, February 2018.

[3] T. Yan, M. Cempini, C.M Oddo and N. Vitiello, Review of Assistive Strategies in Powered Lower-Limb Orthoses and Exoskeletons, Robotics and Autonomous Systems 64, Elsevier, 120-136 , September 2014.

[4] J. Ghan, R. Steger and H. Kazerooni, Control and system identification for the Berkeley lower extremity exoskeleton (BLEEX), Advanced Robotics, Vol. 20, No. 9, 989 - 1014, 2006.

[5] H. Kazerooni, J.L Racine, L. Huang, and R. Steger, On the control of the Berkeley lower extremity exoskeleton (BLEEX), Robotics and automation, ICRA, Proceedings of the 2005 IEEE international conference on on Robotics and Automation, Barcelona, Spain, 4353 - 4360, April 2005.

[6] J.E. Pratt, B.T. Krupp, C. J. Morse and S. H. Collins: The RoboKnee: An Exoskeleton for Enhancing Strength and Endurance During Walking, International Conference on Robotics and Automation, IEEE, New Orleans, LA, 2430 - 2435, April 2004.

[7] A. Ansari, C. G. Atkeson, H. Choset and M. Travers: A Survey of Current Exoskeletons and Their Control Architectures and Algorithms, October 2015.

[8] S. Jezernik, R. G. V. Wassink and T. Keller, Sliding mode closed-loop control of FES: controlling the shank movement, IEEE Transactions on Biomedical Engineering, Vol.51, No.2, 263 - 272, Ferbruary 2004.

[9] J. Figueiredo, C. P. Santos and J. C. Moreno:, Assistance and Rehabilitation of Gait Disorders using Active Lower Limb Orthoses, IEEE 4th Portuguese BioEngineering Meeting, Porto, Portugal, February 2015.

[10] A. Esquenazi, M. Talaty, A. Packel and M. Saulino: The rewalk powered exoskeleton to restore ambulatory function to individuals with thoraciclevel motor-complete spinal cord injury, American Journal of Physical Medicine \& Rehabilitation 91, 911 - 921, 2012.
[11] K. Suzuki, G. Mito, H. Kawamoto, Y. Hasegawa and Y. Sankai: Intention-based walking support for paraplegia patients with robot suit hal, Adv. Robotics 21, 1441-1469, 2007.

[12] S. Mefoued, S. Mohammed and Y. Amirat, Toward Movement Restoration of Knee Joint Using Robust Control of Powered Orthosis,IEEE Transactions on control systems technology, 2156 - 2168, 2013.

[13] S. Mohammed, H. Rifai, W. Hassani and Y. Amirat, Nested saturation based control of an actuated knee joint orthosis, Mechatronics, (23) 8, 1141 - 1149, September 2013.

[14] S. Mohammed, H. Rifai, B. Daachi and Y. Amirat: Adaptive Control of a Human-Driven Knee Joint Orthosis, IEEE International Conference on Robotics and Automation, 2486 - 2491, May 2012.

[15] M. A. Alouane, H. Rifai, Y. Amirat and S. Mohammed Cooperative Control for Knee Joint Flexion-Extension Movement Restoration, IEEE/RSJ International Conference on Intelligent Robots and Systems (IROS), 2018.

[16] K. Sherwani, N. Kumar, A. Chemori, M. Khan, S. Mohammed, RISE-Based Adaptive Control for EICoSI Exoskeleton to Assist Knee Joint Mobility, Robotics and Autonomous Systems (Elsevier), Volume 124, February 2020.

[17] H. Rifai, M-S. Ben Abdessalem, A. Chemori, S. Mohammed and Y. Amirat, Augmented L1 Adaptive Control of an Actuated Knee Joint Exoskeleton: From Design to Real-Time Experiments, IEEE ICRA'16, Stockholm, Sweden, 2016.

[18] B. Maalej, H. Medhaffar, A. Chemori and N. Derbel, A Fuzzy Sliding Mode Controller for Reducing Torques Applied to a Rehabilitation Robot, The 17th International MultiConference on Systems, Signals and Devices - SSD'20, Sfax, Tunisia, 2020.

[19] B. Maalej, A. Chemori and N. Derbel, Intelligent Tuning of Augmented L1 Adaptive Control for Cerebral Palsy Kids Rehabilitation, The 16th International Multi-Conference on Systems, Signals and Devices - SSD'19, Istanbul, Turkey, 2019.

[20] N. Roula, A. Chemori, R. Rizk, Y. Zaatar, On Control Design for a Lower Limb Orthosis: A Comparative Study in Different Operating Conditions, Mechanism, Machine, Robotics and Mechatronics Sciences, Mechanisms and Machine Science (58), pp.81-97, 2019.

[21] L. Martins, C. Cardeira, P. Oliveira, Linear Quadratic Regulator for Trajectory Tracking of a Quadrotor. IFACPapersOnLine, 52(12), 2019.

[22] K. Anurag, S. Kamlu, Design of LQR-PID controller for linearized magnetic levitation system, International Conference on Inventive Systems and Control (ICISC), 2018.

[23] L. M. M. Thwin, Y. Chan, Application of LQR control for two-wheel self-balancing robot, J. Myanmar Acad. Arts Sc, 2020.

[24] A. Malte, R. Patil, Analytical study of PID and LQR: Application to robotic manipulator, International Journal of Advance Scientific Research and Engineering Trends, 2020 . 